\title{
EL ENFOQUE POR COMPETENCIAS DESDE LA PERSPECTIVA DEL DESARROLLO HUMANO. ASPECTOS BÁSICOS Y DISEÑO CURRICULAR
}

\section{The approach for competences from the persepective of the hu- man development. Basic aspects and curricular design}

Héctor E. Martínez Carpio*

\begin{abstract}
Resumen
El artículo propone una visión del enfoque por competencias desde la perspectiva del desarrollo humano. Se inicia con aspectos teóricos sobre desarrollo humano, donde se critica el modelo economicista del PBI y se enfatiza el modelo de calidad de vida propuesto por el PNUD. Como resultado, dentro de los retos de la universidad en el nuevo siglo se propone un concepto de competencia donde se relieva el desarrollo de capacidades y valores para el desarrollo de un proyecto de vida. Profundizando, se explica el significado de, construcción social, aprendizaje significativo, la necesidad de instrumentar la competencia a través de capacidades y valores, la metacognición y el proyecto de vida. Del mismo modo, se plantea finalmente un perfil de egreso del estudiante y una propuesta de planeación curricular, en base a un "programa formativo de asignatura", que permite operacionalizar las capacidades y valores, así como la metodología, y la evaluación por competencias.
\end{abstract}

Palabras clave: Competencia, capacidades, valores, desarrollo humano, proyecto de vida

\begin{abstract}
The article proposes a view of the competence approach from the perspective of human development. It begins with theoretical aspects of human development, where it criticizes the economistic model of GDP and emphasizes the quality model of life proposed by UNDP. As a result, within the challenges of the new century university proposes a concept of competence where it is important to underscore the development of skills and values for the development of a life plan. Looking deeply into explains the meaning of, social construction, significant learning, the need to implement competition through skills and values, metacognition and life project. Similarly, there is finally one student graduate profile and curriculum planning proposal, based on a "course training program" which allows operationalize the skills and values as well as the methodology, and skills assessment.
\end{abstract}

Key words: Competence, skills, values, human development, project of life.

* Psicologo. Director del Centro de Desarrollo Académico (CDA) de la Universidad Católica de Santa María (UCSM)h.mcarpio@yahoo.es 


\section{EL ENFOQUE HUMANISTA DEL DESARRO- LLO HUMANO}

La concepción de desarrollo humano ha sufrido una transformación desde la década del 90 del siglo pasado, intentando generar un cambio radical en el desarrollo de las personas. Inicialmente se relacionó con la producción económica de los países, produciendo como consecuencia el aumento del PBI (producto bruto interno), y por ende mayor desarrollo y satisfacción en la población.

Este enfoque "economicista", donde se enfatiza el PBIcomoelíndice de desarrollo, noexplicalos múltiples fenómenos subjetivos de los grupos poblacionales, donde se insertan fenómenos psíquicos que relativizan aquella ecuación que grafica que, a mayor empleo habrá mayor satisfacción personal. En consecuencia, resulta realmente limitado el enfoque "economicista" del DH., porque, no incorpora fenómenos subjetivos tales como: el proyecto de vida, y fenómenos como los que menciona Morris (1975) (citado por Arteaga, D.) la libertad, la justicia, la felicidad, el tiempo de ocio, la seguridad ciudadana.

Es así que a partir de la intervención de las Naciones Unidas, a través del Programa de las Naciones Unidas para el Desarrollo (PNUD), hoy predomina un enfoque "humanista" del desarrollo humano, que enfatiza la calidad de vida del ser humano, como un fin en sí mismo, generando y ampliando oportunidades para la consecución de una vida digna y feliz. Esta concepción no sólo ha quedado en el "tintero de las oportunidades" muchas veces perdidas y archivadas en monumentales hojas de papel. Sino que se ha incidido en la implementación de políticas en todos los estados del orbe, creándose un factor operacional que se denomina como "índice de desarrollo humano" (IDH), incidiendo su vez, en informes anuales de la evolución del desarrollo humano a nivel mundial.

Pleitez (2001) (citado por Arteaga, D.), considera que dentro de un enfoque "humanista" del desarrollo humano debe observarse "... un tipo de desarrollo que no sólo genera crecimiento económico, sino que también distribuya sus beneficios equitativamente, ejerce un efecto neto positivo en el medio ambiente, y promueve procesos de empoderamiento de la población. Da prioridad a la población pobre, le amplía las capacidades y oportunidades, promueve su participación en los diferentes aspectos que afectan su vida."

Consideramos como factores claves en este proceso, al "empoderamiento" de los sectores poblacionales, porque respeta la particularidad cultural y el desarrollo de capacidades ejerciendo sus derechos y su propia dignidad, en el momento de la elección de sus decisiones. Del mismo modo, como dejamos entrever, el tema de la "ampliación de las oportunidades" como estrategia que los estados deben construir operativamente con el objeto de estimular tanto la autonomía de los individuos (empoderamiento) como el desarrollo de las capacidades.

\section{CONCEPTO DE DESARROLLO HUMANO (DH)}

Desde la perspectiva del desarrollo humano, Amartya Sen economista hindú, conceptúa el desarrollo humano como... la expansión de capacidades, que implica entender la vida humana como formas de ser $y$ hacer (Edo, M. 2002). Esto implica evidentemente, que el desarrollo de capacidades, base operacional de las competencias, cobran sentido si forman parte esencial del ser humano, como manifestación del mundo espiritual del mismo (el ser), que de alguna manera se expresan conductualmente en el hacer.

Dentro del mismo ámbito Tapia, A. (2001), considera que el desarrollo humano es, el constante ejercicio de la capacidad decisoria, libre, y autónoma, orientada a la vida participativa, constructiva, responsable, e innovadora; más la aplicación de los principios éticos, que viabilizan el comportamiento solidario y altruista. Esta concepción vincula evidentementelascapacidades y los valores, subrayando dentro del primer aspecto, la toma de decisiones y el desarrollo autónomo, así como la participación social, vinculados al desarrollo de valores tales como, la responsabilidad, el libre albedrío, la solidaridad y el altruismo.

Esta conceptualización del desarrollo humano, donde intervienen el desarrollo de capacidades y valores útiles para la vida, orientan hacia la construcción de una identidad personal más madura que hacen que la persona actúe de forma competente dentro un mundo 
globalizado. Esto a su vez, nos permite reflexionar sobre la aplicación del enfoque por competencias al ámbito educativo.

\section{DESARROLLO HUMANO Y EDUCACIÓN}

$\mathrm{Si}$ entendemos que la educación permite la expansión de capacidades de los individuos que "construyen" a su vez sus aprendizajes con el apoyo de la información acumulada a lo largo de la historia de la humanidad. El rol de la educación en el desarrollo humano, es de suma importancia.

Es clara nuestra concepción de educación al aplicar el término "construcción de los aprendizajes", que permite la participación activa de la persona en la interpretación del mundo que le rodea; oponiéndose a su vez, a una educación "transmisiva". La experiencia ha demostrado que, no es posible estimular el desarrollo humano con este tipo de instrucción que intenta trasladar los conocimientos hacia los estudiantes que no logran procesar la información, sino que por el contrario imitan conductas o repiten información de forma pasiva y mecánica, sin cuestionar las suposiciones básicas de determinado conocimiento. Y es más, no es posible hablar de una educación contemporánea sino genera conocimiento de acuerdo al nivel de desarrollo del aprendiz, aplicando capacidades superiores relacionadas con el pensamiento crítico, la búsqueda de la innovación a través del pensamiento creativo, la resolución de problemas, y la toma de decisiones.

En suma, una educación que podemos calificarla como "generadora" donde se promueva la expansión de capacidades y el fortalecimiento del desarrollo humano, debe propiciar entornos sugestivos que promuevan el aprendizaje innovador y la capacidad autónoma del estudiante, que se preocupa a su vez por la problemática de su medio social. Esto implica revisar, de acuerdo al contexto donde se desarrolla, su diseño curricular, la metodología, y la evaluación de los aprendizajes, que son momentos concretos de interacción con el estudiante en el salón de clases.

\section{RETOS DE LA EDUCACIÓN SUPERIOR}

Las tendencias mundiales respecto a la Educación Superior se encuentran graficadas por la Declaración
Mundial de la UNESCO sobre la Educación Superior en el Siglo XXI, donde se proclama que las tendencias contemporáneas de la Educación Superior son: la de contribuir al desarrollo sostenible y el progreso del conjunto de la sociedad a través de, formar profesionales altamente cualificados y ciudadanos responsables. Constituir un espacio abierto para la formación superior que propicie el aprendizaje permanente. Promover y difundir conocimientos por medio de la investigación. Contribuir a comprender, interpretar, preservar, reforzar, fomentar y difundir las culturas regionales, nacionales, internacionales. Y contribuir a proteger y consolidar los valores de la sociedad.

Esto implica, que la educación superior contemporánea se orienta por aquél fin educativo que busca formar profesionales competentes, en el ámbito de su especialidad, y ciudadanos responsables. La educación superior es vista no sólo en términos de competencia técnica, que involucra aspectos teórico prácticos, sino también valorativos. La actitud del profesional debe desembocar en una consecuencia social a favor de todas las culturas y estratos sociales. Dentro de esta línea, ante la decreciente durabilidad de los conocimientos, es importante que el profesional desarrolle el aprendizaje autónomo y tenga la convicción que su formación es continua a lo largo de toda la vida.

Las tendencias de la educación superior, desde nuestra perspectiva y de forma específica, las podemos sintetizar de la siguiente manera:

\section{a) Internacionalización del currículo:}

Producto del pasaje de la "sociedad de la información" y el advenimiento de lo que se denomina con mayor frecuencia, como "sociedad del conocimiento", aplicado a la educación superior surge aquella tendencia que pretende la internacionalización del currículo. Sus características se afincan en la propia globalización de las economías y en la necesidad de formar personas capaces de actuar en el ámbito internacional; poniendo en juego sus múltiples visiones del mundo, y recursos cognoscitivos que les permita procesar los datos de forma crítica, emitiendo soluciones innovadoras. Es así que se tiende a conformar espacios y convenios entre países, bloques regionales, 
y universidades, con el propósito de homogenizar las currículas, facilitando el intercambio entre estudiantes y docentes; ampliando a su vez el mercado de trabajo. El Tuning europeo y el de américa latina son dos buenos ejemplos.

b) Generación de conocimiento a través del desarrollo de capacidades superiores:

Las capacidades superiores permiten no sólo captar la información sino transformarla generando conocimiento. Nos referimos específicamente al tipo de pensamiento que se orienta en relación, al pensamiento global, el pensamiento crítico, el pensamiento innovador, y aquellas formas de pensamiento que nos permiten tomar decisiones.

c) La investigación como estrategia pedagógica:

Dentro del trabajo de aula, se tiende hoy en día a aplicar la investigación como estrategia pedagógica, donde a partir de un problema a ser resuelto, el estudiante busca de forma autónoma o cooperativa haciendo uso de bancos de datos especializados o de la aplicación de estrategias técnicas la resolución del problema. Se basa en el aprendizaje por descubrimiento y el cooperativo, así como el aprendizaje basado en problemas. Se busca formar "semilleros de investigación" orientando a los aprendices en la aplicación del método científico, diversificado a lo largo de la carrera profesional. De esta manera surge lo que se denomina hoy en día como "investigación formativa". Aquí el docente de aula acompaña, orienta, y apoya, al estudiante en el reconocimiento y la implementación del método científico en actividades específicas, incentivando así el desarrollo de una cultura investigativa a nivel institucional.

\section{d) Aprendizaje autónomo y continuo:}

Actualmente tenemos que constatar que la figura protagónica del docente ha declinado a favor de la actividad del estudiante. Y es más, si se pretende generar nuevo conocimiento es preciso que el aprendiz desarrolle sus propios medios estratégicos. Una vez que ha aprendido los rudimentos básicos de la materia, el estudiante debe conocer y desarrollar estrategias que favorezcan su propia independencia académica.

e) Énfasis en el fundamento teórico que da sentido a la práctica:
Si bien actualmente existe excesivo énfasis en la actividad que permita la resolución de problemas prácticos en el medio social. Pensamos que se debe regresar a los fundamento teóricos permitiendo el análisis y la evaluación de los fundamentos del quehacer diario al concebir nuevas alternativas de solución y nuevos problemas; rompiendo a su vez con la prácticas rutinarias y mecánicas (el hacer por hacer) del mero profesionista.

f) La universidad como entidad formativa de valores útiles para la vida:

Launiversidadesconceptuadacomounainstitución que no sólo permite la formación profesional sino que es también una entidad formativa, que estimula los valores ciudadanos que permitan desarrollar actitudes relacionadas con la convivencia pacífica y armoniosa con las demás personas. En este sentido, el docente universitario no transmite conocimientos sino que los construye de forma conjunta con los estudiantes. Es esta interacción práctica entre docente y estudiante la que forma personalidades, intercambiando actitudes y valores.

g) Desarrollo de competencias vinculadas a la sociedad:

Como producto de lo anterior, la universidad busca que sus estudiantes sean "competentes" y se vinculen de forma adecuada con la sociedad. Esto implica que sean buenos ciudadanos y buenos profesionales. Ser competente es ser idóneo en lo que se hace, diestros en los fundamentos del quehacer profesional, trasladando los aprendizajes de las aulas universitarias a cualquier situación donde se requiera el uso de la crítica y la innovación, y proyectando su actuación en el futuro, con valores personales, que se actualizan día a día dentro de un proyecto de vida. En el siguiente acápite desarrollamos el análisis sobre este tema.

\section{EL ENFOQUE POR COMPETENCIAS EN LA EDUCACIÓN SUPERIOR}

Son variados los conceptos de competencia desarrollados cada vez con creciente entusiasmo en el ámbito educativo. La sociedad del conocimiento es el marco donde se insertan estas aplicaciones, como un espacio expectante en la historia de la humanidad donde se transforma obligatoriamente la información 
haciendo uso de herramientas mentales integradas de forma compleja, a manera de capacidades, que enfatizan el pensamiento crítico y la creatividad.

$\mathrm{Si}$ bien, todos las concepciones enfatizan los aspectos, cognoscitivos, procedimentales, y actitudinales, como ejes centrales de cualquier enfoque sobre competencias, éstas no deben ser vistas como una sumatoria encadenada y mecánica que desemboca en un resultado (el logro de la competencia). Por el contrario la sociedad contemporánea exige que ésta se analice desde la perspectiva compleja e integral que rompe con la línea de causa y efecto, y que busca dentro de una gama de respuestas el conocimiento pertinente y adecuado para el problema de acuerdo al contexto.

Y es que la competencia no debe ser vista de forma unidimensional obviando sus otros elementos. Por ejemplo, sólo el aspecto procedimental (o aumentando su peso) en detrimento de las dimensiones cognitivas $\mathrm{y}$ valorativas $\mathrm{y}$, sin mediar las cualidades de un determinado problema que se presenta bajo diversas circunstancias sociales. Entendemos que una persona competente analiza estas circunstancias y actúa con un comportamiento pertinente (el más adecuado), con conocimiento fundamentado del problema, con rigor en el ámbito procedimental, y con un conjunto de actitudes que dejan traslucir la convivencia y la armonía con la sociedad.

Es así que, Perrenoud (2004) (citado por Cano, M., 2008), entiende el desarrollo de capacidades en sentido amplio, flexible y creativo, desde una concepción más cercana a la perspectiva cognitiva, más rica y profunda, y que supone entender las competencias como capacidades muy amplias e interconectadas, que implican elegir y movilizar recursos, tanto personales (conocimientos, procedimientos, actitudes) como de redes (bancos de datos, acceso documental, especialistas,...) y realizar con ellos una atribución contextualizada (espacio, tiempo, relación).

Bien sabemos que el tema de las competencias ha sido reeditado con gran amplitud y se ha constituido como una moda, de fácil aplicación a diversas áreas de intervención humana, cuyo origen más reciente se encuentra en el ámbito económico/empresarial.
No obstante, es necesario anotar que vistas así las cosas, esta aplicación se constituye en excesivamente pragmática y utilitaria, porque utiliza al hombre (el desarrollo de conocimientos y habilidades, sobre todo) como un mecanismo a favor de los intereses económicos de la empresa. Con razón Tobón, S. (2008) afirmó lo siguiente... las competencias tienden a ser conceptualizadas de una manera reduccionista y fragmentada, motivo por el cual prima el abordaje de éstas desde la búsqueda de la eficacia y la eficiencia al servicio de intereses económicos.

Aquí es preciso recordar que, etimológicamente competencia proviene de cumpetere, que significa buscar en compañía, o en unión de otros. La palabra tiene raíces en el deporte griego y romano, aunque muy posteriormente pasó a ser utilizado en otros campos y esferas de actuación (Barreto G., Ruiz, J. y Blanco, R. (2008).).

En el ámbito educativo, la aplicación del enfoque por competencias se orienta no sólo a la implementación de un sistema de saberes contextualizados. Donde existe un vínculo estrecho entre conocimiento y habilidad para resolver problemas prácticos dentro del marco social. No obstante, esta concepción de la competencia aplicada a la educación resulta limitada, porque la competencia la excede. La competencia "es más que la capacidad para hacer cosas". Rompe con la idea de que más importante que los saberes de un ser humano es lo que es capaz de hacer con ellos.

Sin embargo, relievando la dimensión conceptual sugerida en el párrafo anterior, no pretendemos que se "traslade" al estudiante un cuerpo de conocimientos con la idea preconcebida que así sabrá fundamentar su actuación fácilmente. La primera atingencia está relacionada con la función generadora del estudiante. Esta función que permite al estudiante construir su propio conocimiento "generando" a su vez nueva información; relativiza la función del docente que pretende trasladar (o transmitir) teoría al estudiante. Por tanto, el producto de la "transmisión" no logra que el estudiante sea competente; por el contrario es un seguidor pasivo.

La segunda atingencia está relacionada con la duración de los contenidos. Aquí es preciso recordar 
que, el énfasis en los contenidos responde al precepto de que se educa para el presente, sin embargo, esos conocimientos enseñados con mucho cuidado quedan obsoletos a los pocos años de ser aprendidos. Por el contrario, el enfoque por competencias aplicado al ámbito educativo, busca la autogestión, la capacidad autónoma e independiente, del aprendiz que relativiza los contenidos, haciéndolos suyos, encontrándoles sentido personal, y transformándolos en nuevo conocimiento. Este es el momento que se desliga de sus propios profesores y materiales de estudio; aplicándolos no sólo a su trabajo profesional sino también a su conducta ciudadana.

Esa es la diferencia sustancial con otras aplicaciones del enfoque por competencias. Desde nuestra perspectiva, en el ámbito educativo el enfoque por competencias busca el desarrollo humano, potencializando las capacidades de la persona ubicada en un contexto social determinado, que actúa con responsabilidad y con valores solidarios, buscando el bien común. Así ser y hacer están unidos al conocimiento, que permite a esta última, reflexionar sobre los fundamentos de las cosas y los fenómenos.

Un elemento es importante destacar, un individuo competente es aquél que puede proyectar hacia el futuro sus capacidades actuales comprendiendo y resolviendo problemas de forma pertinente en aquellos ámbitos proyectados, porque esta persona ha desarrollado mecanismos estratégicos relacionados con el aprender a aprender, desarrollando su autonomía y el sentido personal que le ha permitido mantenerse "a nivel de su tiempo".

Por esos motivos expuestos muy brevemente, nos permitimos proponer el siguiente concepto de competencia, desde la perspectiva del desarrollo humano.

"Es una construcción social, donde se evidencia que la persona ha aprendido de forma significativa. Esto se expresa en el desarrollo de nuevos conocimientos, habilidades, y actitudes; integradas en capacidades y valores, fácilmente transferibles a diferentes situaciones de la vida y al desempeño profesional. Dentro de este proceso son esenciales el desarrollo de capacidades metacognitivas y la perspectiva clara del proyecto de vida de la persona".

\section{La competencia es una construcción social}

Decimos que la competencia es una "construcción social", porque intervienen varios agentes educativos, la institución, el docente, la sociedad, los compañeros de estudio, y el mismo estudiante. Este último agente, es el que "decide", la pertinencia y el sentido de la información para construir sus propias capacidades y valores. Aquí es importante, subrayar el rol que tiene el estudiante en transformar la mera información, que es creciente en el actual momento, en conocimiento. Este proceso es crucial porque los datos son convertidos en base al sentido personal y la interpretación que realiza el individuo, generando conocimiento y nuevas aplicaciones. No podemos pensar que la competencia se logra de pronto de manera intempestiva, como un acto de iluminación del sujeto que intenta resolver problemas. La metáfora de la construcción está presente tanto en el fuero personal como en el externo. Así, el individuo construye sus propios aprendizajes de forma paulatina, e integrando de forma holística, no sumatoria ni mecánica, sus saberes conceptuales, procedimentales, $\mathrm{y}$ actitudinales.

Es importante destacar, como afirma Cano, M. $(2008)^{1}$, que ser competente supone un paso más, que el conocimiento debe ser contextualizado y pertinente. Esto implica, que en el fuero interno el sujeto debe trazar una jerarquía mental de los saberes que son pertinentes para la solución de un problema ubicado en un contexto determinado, e intervenir en el mismo demostrando idoneidad a través de capacidades y valores ajustados a la situación.

Por otro lado, en el fuero externo la dimensión constructiva también se expresa en el momento en que

1. El ser competente implica un paso más: supone, de todo el acervo de conocimiento que uno posee (o al que puede acceder), seleccionar el que resulta pertinente en aquel momento y situación (desestimando otros conocimientos que se tienen pero que no nos ayudan en aquel contexto) para poder resolver el problema o reto que enfrentamos (Cano, M., 2008). 
la institución educativa se convierte en un ambiente facilitadore incitador de aprendizajes, aquílainstitución se organiza de forma intencional, (no espontánea), en estímulo estratégico. Este paso, incluye tanto los agentes educativos (mencionados líneas arriba), como los materiales, y metodologías utilizadas por el docente para facilitar los aprendizajes.

\section{La competencia estimula el aprendizaje significativo}

Efectivamente el desarrollo de competencias estimula el "aprendizaje significativo". A decir de Ausubel, D. (1990), impulsor de este tipo de aprendizaje, es significativo el aprendizaje cuando el nuevo material es relacionado con la experiencia previa del estudiante, elaborando el material de estudio a partir de la estructura cognoscitiva del mismo. Él señaló lo siguiente:

...este proceso (el aprendizaje significativo) reside en que ideas expresadas simbólicamente son relacionadas de modo no arbitrario y sustancial, no al pie de la letra, con lo que el alumno ya sabe... (con una imagen, un símbolo, un concepto, o una proposición). Ausubel, D. (1990, p.48)

Por tanto, el elemento esencial del aprendizaje significativo es "lo que el alumno ya sabe". Ausubel aplicó el término "estructura cognoscitiva". Otros hablaron de: experiencia previa, esquemas cognoscitivos (este último de acuerdo a Piaget). Ausubel decía al respecto:

Si tuviese que reducir toda la psicología de la educación a un solo principio, enunciaría éste: de todos los factores que influyen en el aprendizaje, el más importante consiste en lo que el alumno ya sabe. Averígüese esto, y enséñese consecuentemente. (Ausubel, D., 1990)

Para el Enfoque por Competencias es crucial que la experiencia asuma una estructura que permita un marco de referencia cognoscitivo para los nuevos aprendizajes. Porque de los contrario el individuo no evidenciará competencia a través de sus comportamientos. La estructura cognoscitiva, se expresa de forma jerárquica, cumpliendo la función de organizar la experiencia y de dar sentido a la nueva experiencia. Esto implica que los conceptos previamente aprendidos posibilitarán, en primer lugar, el surgimiento de significados nuevos $y$, en segundo lugar, mejoran la organización y retención de éstos. En consecuencia, de acuerdo a Ausubel; si la estructura cognoscitiva es clara y organizada surgen significados más precisos y disponibles. Si, por el contrario, es inestable y desorganizada tenderá a inhibir el aprendizaje y la retención significativos.

Por ese motivo, dentro del Enfoque por Competencias, es muy importante que el docente averiguie las representaciones mentales de lo que el alumno conoce sobre el tema (estructura cognoscitiva). Y no sólo eso, además debe analizar la dinámica entre esa estructura y los conocimientos nuevos que se quieren enseñar; de tal manera que se puedan programar actividades o procedimientos que faciliten el desarrollo de competencias.

De acuerdo a Ausubel (1990), se pueden considerar como aprendizajes significativos al Aprendizaje significativo por descubrimiento, el cual es un aprendizaje autónomo, basado en la actividad del propio aprendiz, que explora y descubre el mundo, aquí son valiosos los aportes de Jean Piaget y Jerome Bruner. Mientras que por otro lado, se encuentra el Aprendizaje significativo por recepción, denominado también como "aprendizaje por instrucción expositiva". En este último se concentran los aportes de David Ausubel. Es preciso anotar que el Aprendizaje significativo por recepción, denominado también como "aprendizaje por instrucción expositiva", implica un nuevo rol a la exposición del docente, al revalorizar su papel como transmisor de conocimientos; siempre y cuando deje su protagonismo al alumno; y considere que este último tiene una experiencia previa y es constructor de su propio conocimiento. La "exposición" se entiende aquí como "explicación" que intenta establecer las bases para la recepción significativa del nuevo material; este proceso no implica un tipo de aprendizaje que se recepciona mecánicamente utilizando la memoria.

\section{La competencia se integra en capacidades y valores}

Una capacidad la consideramos como una habilidad general, de tipo cognitiva, que se presenta como un potencial; y que involucra aspectos: racional lógico, socio - afectivo, y motor. Evidentemente es una 
concepción cognoscitiva que acentúa el procesamiento de información previo a la acción. También se reconoce que el conjunto de habilidades (específicas) integran una capacidad. También se puede afirmar que una habilidad es una capacidad específica. Del mismo modo, nuestra concepción equipara a la capacidad con las denominadas unidades de competencia (utilizadas por algunos autores). Lo importante es considerar, que el marco general es la competencia, y debajo de ella de forma subordinada se encuentran las capacidades, y luego las habilidades.

Por otro lado, la competencia también incluye un elemento importante dentro del contexto educativo, el desarrollo de valores. El valor es una cualidad de los objetos y de las personas, que los hacen ser valiosos. Su componente principal es afectivo y se expresan en las actitudes. Esta última, la actitud es una disposición hacia la acción, y se expresa en la tonalidad afectiva de aceptación - rechazo, hacia los objetos y personas. Un conjunto de actitudes expresan al valor.

Las competencias se transfieren a diferentes situaciones de la vida y el desempeño profesional

El rol de la transferencia en la educación es muy importante. Se aprende, bajo el supuesto de que lo aprendido se va a transferir automáticamente a todas las situaciones en que actúa el aprendiz. Esto implica que lo aprendido se generaliza al comportamiento de forma natural, alimentando aquella concepción medioeval que afirmaba, que quien sabe, sabe hacer y saber ser.

Esto trajo como consecuencia, que la educación se limitara a enseñar contenidos teóricos con la idea que puede ser transferida fácilmente a los diferentes campos del quehacer humano. Lamentablemente esto no es cierto. El conocimiento de por sí, sobre algo o alguien, no determina el comportamiento práctico y el desarrollo espiritual y personal. Desde hace tiempo esto ha sido cuestionado y aceptado con éxito en los círculos académicos, pero lamentablemente resistido en la práctica del salón de clases.

Zabala, A. y Arnau, L.(2008) grafican claramente esta situación... la presión del saber teórico académico y de las ideas erróneas sobre el aprendizaje y la transferencia de los saberes ha determinado la preponderancia de los conocimientos factuales y conceptuales, tanto es así que para la mayoría del profesorado el término contenidos de enseñanza se limita a los conocimientos...

El enfoque por competencias utiliza una estrategia metodológica integral y globalizadora, donde la presentación de problemas ubicados en el contexto de la vida diaria permite la transferencia del aprendizaje presente del salón de clases, a situaciones futuras que permitan la resolución de problemas fuera de los linderos de la institución educativa. Es evidente, en consecuencia, que la educación debe ser pensada para el futuro, y que debe utilizar variadas metodologías relacionadas con la presentación de situaciones / problema que simulen contextos reales. Además estas metodologías deben orientarse tanto a lo teórico, como al desarrollo de habilidades prácticas y la formación de valores ciudadanos.

\section{Las competencias desarrollan capacidades metacognitivas}

La metacognición es el proceso a través del cual se utiliza los mecanismos cognitivos para tomar conciencia sobre nuestro propio conocimiento. Es el conocimiento del conocimiento. Es el pensamiento sobre el pensamiento. Que permite activar los procesos de autorregulación del funcionamiento cognitivo más específico. Está presente por ejemplo, cuando súbitamente nos damos cuenta de la solución de un problema determinado o cuando tomamos conciencia de las estrategias de estudio para asimilar un determinado material académico, planificando el tiempo de estudio, la técnica a utilizar, los pensamiento distractores que nos asaltan, la evaluación de nuestro desempeño, la identificación de partes del material dificultoso, etc.

En este sentido, la metacognición tiene la meta de perfeccionar el procesamiento de información creando a la vez nuevos productos. Es inevitable pensar que es un componente esencial en la producción de competencias en las personas, si consideramos que el elemento central dentro de este enfoque es el logro de capacidades y valores. Siendo el aspecto cognitivo el elemento previo hacia la acción, que permite a su vez, tomar conciencia de lo que se hace, buscando la calidad y el desarrollo de competencias. 
La metacognición tiene básicamente dos funciones: el autoconocimiento y la autorregulación. En el nivel del autoconocimiento, de acuerdo a Del Buey, F., Martín, Mª, Camarero, F., y Sáez, C., se encuentran los niveles de conocimiento personal, que incluye los aspectos de atribución cognitiva, la motivación, conocimiento del material, estilos de aprendizaje. Mientras que en el conocimiento de la tarea, implica la conciencia de cuánto se conoce sobre el material de estudio, sacando a luz las necesidades de atención y esfuerzo. Finalmente se ubica el conocimiento estratégico, que permite la selección de un plan estratégico para solucionar el problema. En el nivel de la autorregulación se encuentran la planificación, la supervisión, y la evaluación, se trata de un nivel ejecutivo que permite tomar decisiones para la solución de un problema, verificando a su vez los resultados y así lograr la meta propuesta.

Evidentemente, estas dos funciones, el autoconocimiento y la autorregulación convierten a la persona en un sujeto autónomo y competente que construye el conocimiento no como un elemento reactivo sino en base a su propia experiencia, que como hemos visto incluye no sólo el conocimiento de la tarea sino también la concepción estratégica y metodológica para ejecutar la toma de decisiones.

\section{Las competencias favorecen el proyecto de vida de la persona}

Inevitablemente, cuando hablamos de proyecto de vida pensamos en la proyección hacia el futuro de la persona. Un proyecto permite que el sujeto se descentre del momento presente y programe sus acciones hacia el tiempo futuro, planteando sus metas y sus anhelos, junto a los procedimientos para lograr esas metas. Es importante aquí, que la persona se proyecte en base al autoconocimiento y las competencias logradas en el presente, porque éstas le permitirán identificar y reconocer sus aspectos positivos y negativos para lanzarse hacia su vida futura con realismo. Si reconocemos que la calidad del desempeño del sujeto es variable y no asegura el resultado en el futuro, ésta tiene que plantearse dentro de su proyecto de vida, perfeccionando y actualizando sus capacidades, probando las estrategias más eficaces para su consecución.
Siendo el desarrollo humano integral la meta mayor del desarrollo de competencias, el sujeto no sólo debe plantearse como objetivo la satisfacción personal, sino también el compromiso de aportar al desarrollo social. El equilibrio de ambos aspectos, donde el compromiso ético movilizará las herramientas mentales y prácticas que vinculan a la persona con la sociedad, será una demostración fehaciente de que la persona es competente e idónea porque conforma una unidad entre el Ser y el Hacer. Dentro de esta perspectiva el sentido de la vida, así como el sentimiento de pertenencia hacia una colectividad, como el compromiso hacia la misma buscando el bien común con sentido ético, orientan a la persona hacia la autorrealización, no con complacencia ni autocontemplación individualista, sino con la idea de proyectar su vida hacia los diferentes ámbitos del desarrollo humano.

Vistas así las cosas, el enfoque por competencias dentro del ámbito del desarrollo humano aplicado a su vez a la educación nos permite plantear las siguientes metas, que pueden traducirse como un perfil de egreso del estudiante de educación universitaria.

- Actuación ética desde la perspectiva profesional y académica, desarrollando un proyecto de vida que busca la autorrealización y el desarrollo de valores vinculados con el respeto por la verdad, la innovación, la honestidad, y la justicia.

- Procesamiento de la realidad social y personal, en base al desarrollo de habilidades de comunicación oral y escrita en idioma materno y extranjero, y la aplicación racional de las nuevas tecnologías de información y comunicación.

- Profundización en su campo de especialidad, desarrollando capacidades para procesar la información de forma autónoma e independiente, gestionando la información, y generando nuevas respuestas a través de la investigación.

- Capacidad de liderazgo y compromiso social con los sectores sociales menos favorecidos, tomandodecisiones quebusquenaltosestándares de calidad y respeto por el medio geográfico en el desempeño personal y profesional. 
- Valora y respeta la diversidad, considerando el significado de la persona y comprendiendo que las diferencias sociales, culturales, y de capacidades individuales, enriquecen la convivencia y permiten la aproximación hacia la verdad.

\section{EL DESARROLLO DE LAS COMPETENCIAS EN LA UNIVERSIDAD}

El desarrollo de competencias involucra no sólo el trabajo específico dentro del aula, a través de las asignaturas aplicadas directamente hacia los estudiantes. Sino que se necesita de un trabajo previo donde la misma institución juega un rol importante. La aplicación a nivel institucional se inicia de la siguiente manera:

a) Plantear las competencias genéricas del estudiante, a nivel institucional.

Aquí la universidad se hace la siguiente pregunta: ¿qué tipo de estudiante egresará de nuestra casa estudios, luego de haber cursado su carrera profesional?, en otro términos, ¿qué perfil del estudiante exige la institución, de acuerdo a su modelo educativo?. Estas funcionan como competencias genéricas transversales comunes a todas las carreras profesionales.

b) Generar un conjunto de asignaturas que desarrollen las competencias transversales a nivel de toda la universidad.

Un enfoque puro de las competencias no considera la aplicación de asignaturas o cursos, sino que por el contrario lo organiza a manera de módulos de aprendizaje. Se puede aplicar de forma transitoria, un modelo mixto donde las asignaturas responden a un perfil específico de la carrera profesional. Son ejemplo de asignaturas transversales: la Filosofía, Sociología, Psicología, Antropología, Historia, Teología, Propedeútica del Trabajo Intelectual, Comunicación Oral y Escrita, Matemática, Realidad Nacional, Gestión Empresarial, y otras.

c) Describir el perfil de egreso del estudiante por programa profesional, en términos de competencias específicas; organizándolo en un mapa de competencias.
Este aspecto, se encuentra dentro del mismo Programa Profesional que tiene la función de trazar de forma participativa el "perfil de egreso del estudiante" al final de su carrera. En este sentido, Es un perfil integrado que se proyecta a lo que se desea, explicando las funciones y tareas principales que dicha profesión cumple.

d) Seleccionar competencias por áreas especificas o por asignatura.

De acuerdo a las áreas de subespecialidad que pueden existir en cada carrera profesional, o simplemente por asignaturas, se seleccionan competencias consignadas en el perfil. Estas se constituyen en el propósito del área o de la asignatura.

e) Proponer contenidos básicos y bibliografía. Considerando que los contenidos son el medio para lograr las competencias, se seleccionan los contenidos básicos y la correspondiente bibliografía actualizada, incluyendo las referencias digitales.

f) Plantear las estrategias de aprendizaje.

Enseguida, se plantean las estrategias de aprendizaje, como procedimientos y técnicas que permiten el logro de las competencias. Las estrategias más adecuadas para el desarrollo de capacidades en los estudiantes, son aquellas que enfatizan el trabajo mismo del estudiante en intercambio con sus pares, planteando problemas de acuerdo a su nivel de desarrollo. Se recomiendan, las estrategias participativas como: el aprendizaje basado en proyectos, el estudio de caso, la simulación, el aprendizaje in situ, etc.

g) Construcción de la evaluación (¿qué se evaluará? y ¿cuáles son los niveles de exigencia?).

Del mismo modo, siguiendo la línea de construcción de la planificación, este proceso finaliza con la evaluación. Aquí es importante preguntarse ¿qué se evaluará?, ¿con qué técnicas e instrumentos?, ¿qué niveles de exigencia podemos plantear de acuerdo a la experiencia previa de los estudiantes? 


\section{EL DISEÑO DEL PROGRAMA FORMATIVO DE ASIGNATURA}

El "Programa Formativo de Asignatura" es un plan integral y específico de aplicación concreta en el que se formaliza las actividades de aprendizaje que se realizarán para que los estudiantes desarrollen competencias, en concordancia con el perfil de egreso de la carrera profesional desde la perspectiva de la asignatura. Este documento, permite la labor interna del docente dentro de la sesión de aprendizaje. Es una guía de conducción que orienta y organiza las sesiones de aprendizaje; permite la planificación pedagógica, que facilita el aprendizaje/ enseñanza.

Este Programa Formativo consigna de forma detallada, las "competencias" por cada fase de aprendizaje, las "unidades de competencia", los "temas", las "estrategias de aprendizaje/enseñanza", y la "evaluación de los aprendizajes", en base a evidencias y criterios de evaluación. Enseguida describimos el significado de cada una de ellas.

- Competencia: es el propósito del aprendizaje, se deben considerar las tres dimensiones de la competencia: saber conceptual, saber procedimental, y saber actitudinal. Así mismo, la siguiente estructura: verbo indicativo + objeto + condición.

- Las unidades de competencia: son las "capacidades" que las podemos definir como, el conjunto de habilidades y destrezas que desarrolla la persona a lo largo de la vida, producto de la experiencia y la educación. Se expresan a través de procesos cognitivos, sociales, afectivos, y motrices. Se organizan a través de los siguientes saberes:

- El saber conceptual: su contenido se enfoca en el aspecto cognitivo y teórico, con la finalidad de crear conocimiento fundamentado en los estudiantes. Aquí son importantes el desarrollo del "pensamiento crítico" y el "pensamiento creativo", como capacidades superiores que implican, el análisis, la inferencia, el enjuiciamiento, la argumentación, la crítica, etc.
- El saber procedimental: tiene la función de aplicar el conocimiento construido por el estudiante, como un conjunto de métodos y procedimientos para resolver problemas. Aquí es importante desarrollar tanto el "pensamiento resolutivo", como el "pensamiento ejecutivo", que implican, el análisis de problemas prácticos, el control de procesos, la clasificación, la investigación, etc.

- El saber actitudinal: tiene la función de estimar, apreciar, el conocimiento y las aplicaciones prácticas de la asignatura. Son los valores expresados a través de las actitudes, como disposiciones que preparan para la acción. Son importantes aquí los valores de: responsabilidad, la sinceridad, la autoestima; expresados en actitudes como: la puntualidad, el respeto a normas, el compromiso, el liderazgo, el respeto por el otro, la coherencia, la aceptación del otro, el deseo de superarse, etc.

- Temas de unidad: Se trata de contenidos conceptuales básicos que serán desarrollados en cada unidad. Estos contenidos se constituyen en medios y están subordinados a las capacidades.

- Estrategias de Aprendizaje/Enseñanza: las estrategias de aprendizaje describen las actividades que realizará el estudiante, ya sea desde dentro del aula como fuera de ella. En consecuencia, es necesario mencionar incluso las actividades de preparación evidenciadas a través de un producto que luego serán presentadas al docente; así como las actividades presenciales.

- La evaluación de los aprendizajes: considera tanto la presentación de "evidencias" como la confección de los "criterios" que permitirán la evaluación objetiva de las capacidades adquiridas del aprendiz. Consideramos tres tipos de evidencia: "evidencias de conocimiento", "evidencias de desempeño" y "evidencias de producto". Son ejemplos de evidencias de 
conocimiento: los exámenes escrito y oral. De evidencias de desempeño: informe de prácticas, reporte de las prácticas por parte de personal a cargo. De evidencias de producto: una maqueta, un proyecto, un ensayo, una monografía, etc.

Respecto a los "criterios de evaluación", trata de las características de la evidencia que especifica incluso, el estándar mínimo de calidad de las evidencias presentadas por el estudiante para acreditar que ha adquirido las competencias. El estándar mínimo de calidad es expresado a través de indicadores de logro. Estas pueden ser entendidas como capacidades específicas que debe demostrar el estudiante para ser "aprobado"; por lo que podemos considerar de que se trata de conductas cualitativas externas y observables, susceptibles de ser cuantificadas. Debiendo ser logrados a través de evidencias. Son ejemplos de criterios de evaluación, las características de una monografía señalando el estándar mínimo de logro. Por ejemplo, 05 referencias bibliográficas con una antigüedad de menos de 03 años, discusión grupal del tema fundamentando las ideas en 03 carillas como mínimo, desarrollo de marco conceptual describiendo las categorías fundamentales y respetando las normas de cita, agregando además el criterio fundamentado de los autores de la monografía, etc.

- Actividades de Investigación Formativa, Proyección Social, y Extensión Universitaria: tiene el propósito de familiarizar al estudiante con, aspectos, actividades específicas, que se encuentran dentro del proceso de investigación, proyección, y extensión. En consecuencia, no se trata de realizar investigaciones completas y rigurosas, sino de descomponer el proceso en aspectos esenciales que permitan al estudiante "formarse" y "prepararse", para actividades de mayor rigor. Por ejemplo, familiarizarse con buscadores digitales especializados, formular problemas de investigación, estructurar las monografías con las partes de una investigación, implementar programas de intervención, diagnóstico de problemas de una comunidad, interpretación de cuadros estadísticos, etc.

En el anexo 1 graficamos un formato operativo de planificación del "programa formativo de asignatura", que se viene aplicando en la Universidad Católica de
Santa María de Arequipa, Perú; elaborando por el autor como miembro integrante del Centro de Desarrollo Académico de la mencionada universidad.

\section{CONCLUSIONES:}

- Contrario al modelo de competencias aplicado al ámbito empresarial, existe una diferencia significativa en la aplicación del enfoque por competencias en el ámbito educativo. Esta última busca mejorar la calidad de vida de las personas a través del aprendizaje, permitiendo el desarrollo humano.

- El desarrollo humano aplicado a las competencias, permite valorarlo, de acuerdo al PNUD, como una ampliación de capacidades y oportunidades en el ser humano. Lo que trae como consecuencia la responsabilidad, no sólo del individuo de desarrollar capacidades; sino también de la misma sociedad, a través de sus instituciones, quienes deben crear el ambiente propicio para el desarrollo personal.

- El enfoque por competencias desde la perspectiva del desarrollo humano, concibe al aprendizaje como una construcción de las siguientes dimensiones: saber conceptual, saber procedimental, saber actitudinal. Lo que se constituye en un enfoque complejo que busca el desarrollo integral y equilibrado de la persona.

- Es importante la planificación, creando estrategias e instrumentos específicos, para el desarrollo de competencias en una organización universitaria. Esta se inicia desde el diseño del plan formativo institucional, donde participan todos los agentes educativos; hasta la elaboración del "programa formativo de asignatura" donde se planifica la instrucción pedagógica. 


\section{REFERENCIAS}

Arteaga, D. Desarrollo humano y generación de capacidades.En:http://www.udb.edu.sv/dialogos/ PDF/ArtD5_4.pdf. Recuperado 16/02/2013.

Ausubel, D. (1990). Psicología educativa. Un punto de vista cognoscitivo. México: Trillas. 9na. Reimpresión.

Barreto G., Ruiz, J. y Blanco, R. (2008). En Revista Iberoamericana de Educación ISSN: 1681$5653 n .^{\circ}$ 45/1. EDITA: Organización de Estados Iberoamericanos para la Educación, la Ciencia y la Cultura (OEI).

Cano, M. (2008). La evaluación por competencias en la educación superior. Barcelona. http://www.ugr. es/local/recfpro/rev123COL1.pdf. Recuperado el 27 de Junio, 2010.

Conferencia Mundial sobre la Educación Superior (octubre de 1998). Declaración Mundial sobre la Educación Superior en el Siglo XXI: visión y acción. Marco de acción prioritaria para el cambio y el desarrollo de la educación superior: UNESCO, París. http://www.unesco.org/ education/educprog/wche/declaration_spa.htm. Recuperado: 02/02/013.
Del Buey, F., Martín, Mª , Camarero, F., y Sáez, C. Procesos metacognitivos: estrategias y técnicas. http://www.profes.net/rep_documentos/ Monograf/1PEI_ProcMetacognitivos_b.pdf. Recuperado el 04-08-2011

Edo, M. (2002). El desarrollo como libertad. La viabilidad de una alternativa a las estrategias de promoción del desarrollo. www.stp.gov. py $/$ ?module=file $\&$ act $=$ procFileDownload...srl... Recuperado: 12/02/013

Martínez, H., Cuentas, T., Zanabria, H., Benavente, A., y Cardeña, M. (2012). El desarrollo de competencias en la formación universitaria. Guía de diseño de la instrucción del docente de la U.C.S.M. Arequipa: Centro de Desarrollo Académico.

Tobón, S. (2008). Formación basada en competencias. Colombia: ECOE. 2da. Ed.

Tapia, A. (2001). Educación y Desarrollo Humano. Arequipa.

Zabala, A. y Arnau, L.(2008). Cómo aprender y enseñar competencias. España Ed. Graó.

Fecha de recepción: 15 de abril de 2013

Fecha de aceptación: 25 de mayo de 2013 


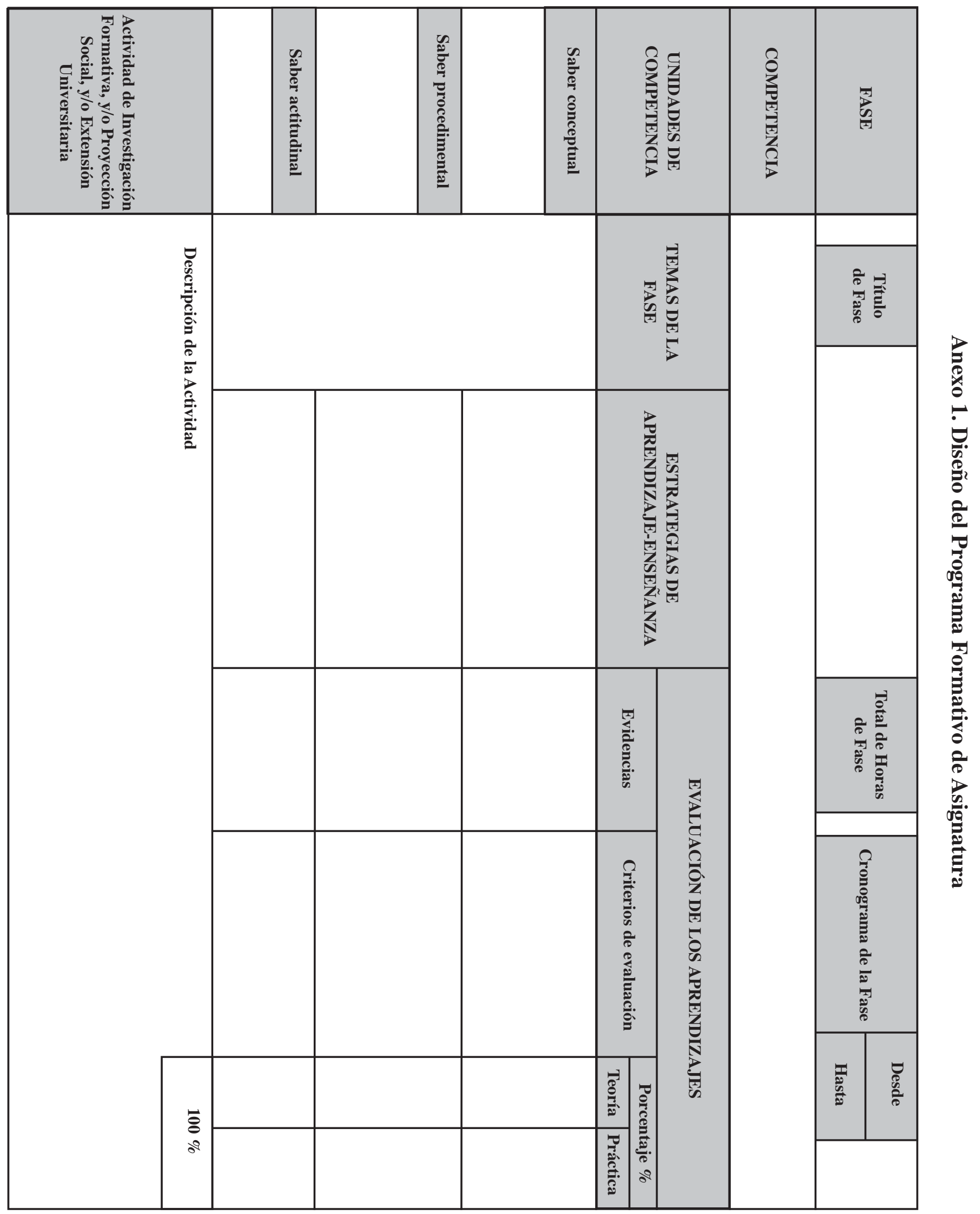

